\title{
Enquête ethnobotanique et criblage phytochimique de quelques plantes tinctoriales du Niger en vue d'une valorisation en énergie solaire
}

\author{
Moutari SOULEY KALLO ${ }^{1}$, Rabani ADAMOU ${ }^{1 *}$, Jacques SAWADOGO ${ }^{2}$, \\ Abdoulkadri AYOUBA MAHAMANE ${ }^{1}$, Inoussa MAMAN MAAROUHI ${ }^{3}$ \\ et Khalid IKHIRI ${ }^{1}$
}
${ }^{1}$ Laboratoire Matériaux, Eaux et Environnement (LAMEE), Groupe de Recherche Physico-Chimie des Matériaux et Photochimie Appliquée, Département de Chimie, Faculté des Sciences et Techniques, Université Abdou Moumouni. BP 10667, Niamey, Niger.
${ }^{2}$ Laboratoire Sol - Eau - Plante, Département de Gestion des Ressources Naturelles et Systèmes de Productions, Centre National de la Recherche Scientifique et Technologique (CNRST), 01 BP 476 Ouagadougou 01, Burkina Faso.
${ }^{3}$ Laboratoire Garba Mounkaila, Département de Biologie, Faculté des Sciences et Techniques, Université Abdou Moumouni. BP 10667, Niamey, Niger.
*Auteur correspondent, E-mail : rabadamou@gmail.com /Tel: + 22790238746

\section{REMERCIEMENTS}

Nos sincères remerciements vont au Ministère fédéral de l'éducation et de la recherche allemand $(B M B F)$ et au Centre des services scientifiques de l'Afrique de l'Ouest sur les changements climatiques et l'utilisation des terres adaptées (WASCAL) pour leur bourse partielle.

\section{RESUME}

Les cellules solaires à pigments photosensibles semblent être le dispositif photovoltaïque d'avenir. Toutefois, la toxicité et le coût de synthèse des pigments limitent l'utilisation accrue desdits prototypes. Les pigments naturels végétaux peuvent constituer une alternative efficace, durable et bon marché. Dans cette étude, l'enquête ethnobotanique des plantes traditionnellement utilisées dans la teinture (textile, maroquinerie, poterie et esthétique) a été réalisée auprès des herboristes, des artisans teinturiers, des chercheurs nigériens et des tradipraticiens de la région de Niamey. Par la suite, le criblage phytochimique, via des réactions colorées et/ou de précipitations de vingt plantes les plus citées, a été réalisé. L'enquête ethnobotanique a permis de répertorier une quarantaine d'espèces végétales qui sont utilisées dans la production des colorants. Ces plantes sont réparties dans 23 familles dont les plus dominantes (62,5\% des espèces recensées) sont les Caesalpiniaceae (12,5\%), les Combretaceae (10\%), les Fabaceae (10\%), les Anacardiaceae (10\%), les Poaceae (5\%), les Cochlospermaceae (5\%), les Méliaceae (5\%) et les Mimosaceae (5\%). Des tannoïdes, flavonoïdes, anthocyanes, quinones, coumarines, alcaloïdes, stérols et polyterpènes sont détectés dans les extraits de ces plantes. L'abondance remarquable des diverses molécules colorantes fait de ces plantes des matières premières potentielles pour la production des colorants naturels valorisables en énergie solaire, particulièrement dans les cellules solaires à pigments photosensibles.

(C) 2018 International Formulae Group. All rights reserved.

Mots clés: Inventaire des plantes, pigments naturels, test phytochimique, énergie solaire, Niger. 


\title{
Ethnobotanical survey and phytochemical screening of some Niger dye plants for their use in solar energy
}

\begin{abstract}
Dye sensitized solar cell appear to be the future photovoltaic device. However, the toxicity and the cost of synthetic pigments limit the development of these prototypes. Natural pigments can be an effective, sustainable and inexpensive alternative. In this study, the ethnobotanical survey of plants traditionally used in dyeing (textiles, leather goods, pottery and aesthetics) was carried out among herbalists, dyeing craftsmen, researchers and traditional healers in the Niamey region. Subsequently, phytochemical screening, through colored and / or precipitation reactions of twenty of these most quoted plants was carried out. The obtained results allowed identifying forty (40) plant species that are used in the production of dyes. These plants are distributed in 23 families, including Caesalpiniaceae (12.5\%), Combretaceae (10\%), Fabaceae (10\%), Anacardiaceae (10\%), Poaceae, Cochlospermaceae, Meliaceae and Mimosaceae, 5\% each. Tannoids, flavonoids, anthocyanins, quinones, coumarins, alkaloids, sterols and polyterpenes are detected in these plants extracts. The remarkable abundance of the various coloring molecules makes these plants potential raw materials for the production of natural dyes that can be used in solar energy.
\end{abstract}

(C) 2018 International Formulae Group. All rights reserved.

Keywords: Inventory of plants, natural dye, phytochemical test, solar energy, Niger.

\section{INTRODUCTION}

Depuis très longtemps, les colorants et pigments naturels étaient utilisés à travers le monde par les populations pour teindre les célèbres tapisseries dans les textiles (Cardon et al., 2010) et colorer les produits alimentaires (Macfoy, 2004). Toutefois, l'expansion de la chimie et le développement de la chimie de synthèse au début du XX ${ }^{\text {ème }}$ siècle ont permis la production des pigments synthétiques à grande échelle, lesquels étaient considérés comme plus fiables, de qualité constante, ayant une plus grande intensité de couleurs et plus stables (Macfoy, 2004). Cela a réduit considérablement l'utilisation des colorants naturels. Malheureusement, les colorants synthétiques ne sont pas sans conséquences pour l'homme et l'environnement, ce qui a conduit à avoir recours, une fois de plus, aux pigments naturels.

En effet, au-delà des pigments organiques de synthèse, les pigments naturels constituent une alternative de premier choix et leur utilisation s'inscrit dans une démarche de développement durable. En outre, ces derniers sont exempts de métaux, non toxiques, non cancérigènes, largement accessibles, disponibles à très faible coût et biodégradables naturellement sans aucune autre forme de pollution (Siva, 2007; Sore et al., 2012; Mabika et al., 2013; Boye et al., 2014). Aujourd'hui, leur domaine d'utilisation s'étend dans la production de l'énergie, notamment en énergie solaire où ils assurent la collection de la lumière du soleil et sa conversion en électricité à l'aide des cellules solaires à pigments photosensibles. Par ailleurs, les pigments assurent l'injection des électrons dans la bande de conduction des semi-conducteurs à une vitesse ultrarapide lorsqu'ils sont excités avec de la lumière visible (Calogero et al., 2012). De plus, ils possèdent suffisamment de groupes hydroxyles leur permettant de se lier à la nanostructure du semi-conducteur et une bonne efficacité à collecter une grande partie des radiations solaires (Yamazaki et al., 2007; Narayan, 2012). Toutes ces propriétés en font de bons candidats pour les cellules solaires à pigments photosensibles.

De nombreuses études sur des cellules sensibilisées avec des pigments naturels ont été effectuées et des rendements de conversion appréciable ont été obtenus. Calogero et al. (2012) ont montré que des cellules sensibilisées avec des anthocyanines et betalaines naturels avaient fourni un 
rendement de conversion de l'ordre de $2,06 \%$. Par ailleurs, un maximum de conversion de 1'ordre de $2,87 \%$ a été obtenu avec le pigment de Kenaf Hibiscus (Saha et al., 2016). En Afrique, les plantes, particulièrement celles tinctoriales constituent une importante source de phytocomposés qui sont utilisées pour la production de couleurs en tannerie, en vannerie et dans le textile (Siva, 2007; Sore et al., 2012; Mabika et al., 2013; Tutak et al., 2014; Fagbohoun, 2014), mais aussi en médecine traditionnelle pour leurs propriétés thérapeutiques (Sofowora, 2010; Zerbo et al., 2012; Odeja et al., 2014; Koffi et al., 2015; Béné et al., 2016). Ainsi, il a été rapporté que certaines plantes produisaient des colorants possédant généralement des propriétés tinctoriales souhaitables qui sont comparables à celles de certains colorants synthétiques hautement appréciés (Siva, 2007). Par ailleurs, d'importants travaux ont été effectués afin d'évaluer la phytochimie, l'activité biologique et pharmacologique de certaines plantes tinctoriales (Kini et al., 2008; Millogo-Kone et al., 2008; Udobi et al., 2009; Bakasso, 2009; Badiaga, 2011; Gnahoué et al., 2015). Au Niger, le domaine des colorants naturels n'a pas été suffisamment exploré. Il reste toujours un domaine vierge nonobstant quelques utilisations traditionnelles de Lawsonia inermis L. en cosmétique, de Indigofera tinctoria $\mathrm{L}$. en textile, de Acacia nilotica en tannerie, du Sorghum bicolor en vannerie et de Hibiscus sabdarifa comme colorant et ingrédient alimentaire. Il est donc nécessaire d'entreprendre des études ethnobotaniques et phytochimiques expliquant les utilisations locales des espèces végétales tinctoriales du Niger. La présente étude visait à mener une enquête ethnobotanique des plantes traditionnellement utilisées dans la teinture (textile, maroquinerie, poterie et esthétique) auprès des herboristes, des artisans teinturiers, des chercheurs nigériens et des tradipraticiens de la région de Niamey et à réaliser un criblage phytochimique afin de détecter d'éventuels pigments responsables des couleurs observées. Elle constitue une première initiative de valorisation des plantes tinctoriales du Niger et de la sous-région en énergie solaire.

\section{MATERIEL ET METHODES}

\section{Matériel}

\section{Matériel végétal}

Le matériel végétal est constitué de diverses parties dont les écorces, racines, feuilles, fleurs, fruits et noix de vingt (20) plantes les plus citées au cours des enquêtes dans la région de Niamey. Il s'agit de l'écorce de tronc de Lannea microcarpa Engl. et K. Krause (Anacardiaceae), Sclerocarya birrea (A. Rich) Höchst (Anacardiaceae), Khaya senegalensis (Desv.) A. Juss (Méliaceae), Detarium microcarpum Guill. et Perr. (Caesalpiniaceae), Parkia biglobosa (Jacq.) Benth (Mimosaceae), Commiphora africana (A. Rich) Engl (burseraceae), des feuilles d'Anogeissus leiocarpus (DC.) Guill. et Perr. (Combretaceae) et de sorghum spp (Poaceae), des parties aériennes de Striga hermontheca (Del.) Benth (Scrofulariaceae) et Indigofera tinctoria L (Fabaceae), des fleurs de Hibiscus sabdarifa L. (Malvaceae), des racines de Sarcocephalus latifolia (Sm.) Bruce et Cochlospermum planchonii hook (Cochlospermaceae) (Tableau 3). Ces plantes ont été fraîchement collectées et identifiées au Département de Biologie de la Faculté des Sciences et Techniques de l'Université Abdou Moumouni de Niamey. Des spécimens ont été déposés à l'herbier dudit Département. Les échantillons étudiés ont été d'abord lavés, séchées au laboratoire en absence de la lumière (dans l'étuve à $50{ }^{\circ} \mathrm{C}$ ) et broyées sous forme de poudre qui par la suite, a été utilisée pour effectuer les tests.

\section{Matériels techniques}

Les échantillons récoltés ont été séchés dans une étuve à $50{ }^{\circ} \mathrm{C}$ avant leur pulvérisation grâce à un broyeur manuel pour obtenir des poudres fines. Une balance électrique pour les différentes pesées, du 
coton hydrophile et des papiers Wattman $\mathrm{N}^{\circ} 1$ ont été utilisés comme filtre. Un évaporateur rotatif (Rotavapor R-III - Buchi) a été utilisé pour concentrer les extraits.

\section{Réactifs et solvants}

Les réactifs utilisés pour le criblage phytochimique sont consignés dans le Tableau 1. L'éther de pétrole (pour la macération), l'eau distillée (pour la décoction), l'éthanol et le méthanol ont été utilisés pour la préparation des extraits. Tous les réactifs et les solvants utilisés sont de qualité analytique.

\section{Méthodes}

\section{Enquête ethnobotanique}

Une enquête ethnobotanique sur les plantes tinctoriales du Niger a été menée dans la région de Niamey auprès des herboristes, des artisans teinturiers, des chercheurs nigériens et des tradipraticiens. Une fiche d'enquête ethnobotanique renfermant un questionnaire a été proposé. Afin de répondre au questionnaire, des interviews directes entre deux personnes dont l'une transmet à l'autre des informations ont été effectuées (Mabika et al., 2013; Fagbohoun, 2014; Koffi et al., 2015). Le but de cette enquête est d'obtenir des renseignements non seulement sur la nature, le type et la disponibilité desdites plantes, mais aussi sur les parties utilisées et les techniques d'extraction des colorants. Les sites d'enquête de la zone d'étude ont été choisis non seulement pour leurs climats qui regorgent pratiquement de toutes les espèces végétales du pays, mais aussi de l'importance accordée à l'usage des plantes tinctoriales et à la pharmacopée traditionnelle nigérienne.

\section{Criblage phytochimique}

Le criblage a été réalisé sur les vingt (20) plantes (Tableau 3) les plus citées dans le domaine tinctorial. Les tests menés visent à mettre en évidence la présence des principaux pigments (caroténoïdes, anthocyanes, flavonoïdes...) responsables de l'apparition de couleur chez les végétaux supérieurs et d'autres substances pouvant contribuer à l'amélioration de la collecte des radiations lumineuses. Les composés ciblés constituent les tannoïdes, les flavonoïdes, les anthocyanes, les tanins, les quinones, les leuco anthocyanes, les alcaloïdes, les coumarines, les stérols et polyterpènes. Le screening a été réalisé au moyen des tests de caractérisation phytochimique via des réactions de coloration ou de précipitations sur les extraits qui sont décrits par certains auteurs (Dohou et al., 2003; Bekro et al., 2007; N'Guessan et al., 2009; Campêlo et al., 2011; Alilou et al., 2014; Odeja et al., 2014; Koffi et al., 2015).

\section{Détection des alcaloüdes}

Les alcaloïdes sont des composés organiques azotés pratiquement d'intérêt thérapeutique. Des alcaloïdes colorés en jaune (Młodzińska, 2009; Mabika et al., 2013), bleu et rouge (Młodzińska, 2009) ont été isolés des plantes. Leur mise en évidence a été effectuée à l'aide de réactif de Dragendorff (réactif à l'iodo-bismuthate), de Mayer (réactif à iodo-mercurate) et de Wagner (réactif iodo-ioduré) par les procédures décrites dans des travaux antérieurs (Razafindrambao, 1973; N'Guessan et al., 2009; Sangare et al., 2012; Koffi et al., 2015).

\section{Détection des polyterpènes et stérols}

Les polyterpènes constituent un vaste groupe de composés terpéniques, dont les caroténoïdes composés des carotènes, des xanthophylles et des acides caroténoïques; pigments jaunes, oranges, rouges retrouvés chez les végétaux. Les polyterpènes et stérols ont été mis en évidence dans les résidus par la réaction de Liebermann (Koffi et al., 2015). Ainsi, $5 \mathrm{~g}$ de la poudre sont macérés dans 20 $\mathrm{ml}$ d'éther de pétrole pendant $24 \mathrm{H}$. Après filtration et évaporation à sec à $90{ }^{\circ} \mathrm{C}$, le résidu est dissout à chaud dans $1 \mathrm{ml}$ d'anhydride acétique, puis reprise dans un tube à essai dans lequel sont coulés $0,5 \mathrm{ml}$ de $\mathrm{H}_{2} \mathrm{SO}_{4}$ concentré. L'apparition d'une 
coloration violette qui vire au bleu puis au vert indique une réaction positive

\section{Détection des flavonoüdes}

Les flavonoïdes sont des composés phénoliques ou aromatiques responsables des colorations jaune et orange de nombreuses fleurs, de fruits et parfois de feuilles jeunes sénescentes (Koko et al., 2011; Narayan, 2012). Les flavonoïdes ont été mis en évidence par le test de Shibata (Ciulei, 1982; Bekro et al., 2007; Koffi et al., 2015). Ainsi, $2 \mathrm{ml}$ de décocté sont évaporés à sec et le résidu obtenu est dissout dans $2 \mathrm{ml}$ de méthanol $50 \%$ puis transvasé dans un tube à essai. Des fragments de tournure de magnésium et 4 gouttes de $\mathrm{HCl}$ concentré ont été ajoutés dans le tube. L'apparition d'une coloration rouge ou orange indique la présence d'aglycones de flavonoïdes. L'addition de 2 à 3 gouttes d'alcool iso amylique a intensifié cette coloration qui a confirmé la présence de flavonoïdes.

\section{Détection des anthocyanes}

Les anthocyanes constituent une classe de flavonoïdes responsables de la coloration rouge et violette de nombreux fruits, fleurs et certaines feuilles. Un large répertoire de couleurs dans la gamme rouge bleu est disponible pour les anthocyanines en raison de leur complexation avec d'autres polyphénols et ions métalliques. Les anthocyanes ont été mises en évidence via le protocole suivant : $2,5 \mathrm{ml}$ du décocté aqueux sont ajoutés à $1 \mathrm{ml}$ de $\mathrm{HCl}$ à $20 \%$, une coloration rose- rouge s'accentue; puis l'ajout de $5 \mathrm{ml}$ de $\mathrm{NH}_{4} \mathrm{OH}$ fait virer la solution au bleu violacé indiquant ainsi la présence d'anthocyanes (Badiaga, 2011).

\section{Détection des leuco anthocyanes}

Ici, $5 \mathrm{ml}$ de décocté est mélangé à 4 $\mathrm{ml}$ d'alcool chlorhydrique (Éthanol/ $\mathrm{HCl}$ pur $3 / 1 \mathrm{v} / \mathrm{v}$ ). Après chauffage au bain-marie à 50 ${ }^{\circ} \mathrm{C}$ pendant quelques minutes, l'apparition d'une couleur rouge cerise indique la présence des leuco anthocyanes (Razafindrambao, 1973).

\section{Détection des tannoïdes}

Les tannoïdes (tannins hydrolysables) et les tannins vrais (condensés) sont des polymères de polyphénols responsables de colorations noires brunes des extraits végétaux. Ils ont été détectés en ajoutant quelques gouttes de solution de $\mathrm{FeCl}_{3}$ diluée à 1 ou $2 \%$ au décocté. L'apparition d'une coloration verdâtre indique la présence des tanins catéchiques ou bleu noirâtre pour les tanins galliques (Bekro et al., 2007; Alilou et al., 2014; Koffi et al., 2015).

\section{Détection des tannins condensés}

Ces tanins ont été mis en évidence à chaud en présence de $\mathrm{HCl}$ concentré. À $2 \mathrm{ml}$ d'extrait sont ajoutées quelques gouttes de $\mathrm{HCl}$ concentré, le tout est chauffé au bainmarie bouillant. La formation d'un précipité rouge indique un test positif (Bekro et al., 2007)

\section{Détection des quinones}

Les quinones sont des composés organiques dont certains sont des pigments jaune, orange, violet et rouge retrouvés chez les plantes. Ces substances ont été recherchées via le réactif de Bornstraëgen (Bekro et al., 2007; N'Guessan et al., 2009; Koffi et al., 2015). Par ailleurs, elles ont été également recherchées dans l'extrait de l'éther de pétrole par ajout de quelques gouttes de $\mathrm{NaOH} 10 \%$. L'apparition d'une teinte qui vire au jaune, rouge ou violet dans la phase aqueuse indique une réaction positive (Dohou et al., 2003)

\section{Détection des coumarines}

Les coumarines ont été mises en évidence par la procédure décrite par Békro et al. (2007). En effet, à $2 \mathrm{ml}$ de solution éthanoïque est ajouté $0,5 \mathrm{ml}$ de $\mathrm{NaOH}$ à $10 \%$. Après chauffage et refroidissement, $4 \mathrm{ml}$ d'eau distillée sont rajoutés. La solution devient transparente par rapport au témoin. La réaction est positive si l'acidification de la solution transparente avec quelques gouttes de $\mathrm{HCl}$ concentré fait perdre sa coloration jaune pour la rendre trouble ou s'il se forme un précipité 
Tableau 1: Réactifs utilisés dans le criblage phytochimique.

\begin{tabular}{|c|c|c|c|c|c|c|c|c|c|c|}
\hline $\begin{array}{l}\text { Groupe } \\
\text { chimique }\end{array}$ & Alcaloïdes & Flavonoïdes & $\begin{array}{c}\text { stérols et poly } \\
\text { terpènes }\end{array}$ & Quinones & Anthocyanes & Tannoïdes & $\begin{array}{c}\text { Tanins } \\
\text { vrais }\end{array}$ & $\begin{array}{c}\text { Couma } \\
\text { rines }\end{array}$ & $\begin{array}{c}\text { Leuco antho- } \\
\text { cyanes }\end{array}$ & Anthocyanes \\
\hline $\begin{array}{l}\text { Réactifs } \\
\text { d'identifica } \\
\text { tion }\end{array}$ & $\begin{array}{l}\text { Réactif de } \\
\text { Mayer; } \\
\text { Réactif de } \\
\text { Dragendorff; } \\
\text { Réactif } \\
\text { Wagner }\end{array}$ & $\begin{array}{c}\text { Acide } \\
\text { chlorhydrique; } \\
\text { Ammoniaque } \\
\text { Copaux de } \\
\text { Zinc }\end{array}$ & $\begin{array}{c}\text { Acide } \\
\text { sulfurique } \\
\text { concentré ; } \\
\text { Anhydride } \\
\text { acétique }\end{array}$ & $\begin{array}{c}\text { Acide } \\
\text { chlorhydrique } \\
\text { au } 20 \% \\
\text { Ammoniaque } \\
\text { concentrée } \\
50 \%\end{array}$ & $\begin{array}{l}\mathrm{H}_{2} \mathrm{SO}_{4} \text { à } 10 \%, \\
\text { Ammoniaque }\end{array}$ & $\begin{array}{c}\mathrm{FeCl}_{3} \\
\text { diluée à } \\
2 \% .\end{array}$ & $\begin{array}{c}\mathrm{HCl} \\
\text { concentré }\end{array}$ & $\begin{array}{l}\mathrm{NaOH} \\
\text { à } 10 \% ; \\
\mathrm{HCl} \\
\text { concent } \\
\text { ré }\end{array}$ & $\begin{array}{c}\text { Éthanol / HCl } \\
\text { pur 3/1 v/v }\end{array}$ & $\begin{array}{l}\mathrm{H}_{2} \mathrm{SO}_{4} \text { à } 10 \%, \\
\text { Ammoniaque }\end{array}$ \\
\hline
\end{tabular}




\section{RESULTATS \\ Étude ethnobotanique \\ Plantes tinctoriales recensées et leurs indications dans la production de couleurs}

L'enquête ethnobotanique effectuée dans la région de Niamey a permis de répertorier une quarantaine d'espèces végétales réparties dans 23 familles. Le Tableau 2 présente les différentes espèces de plantes enregistrées au cours de l'enquête ethnobotanique, leur mode d'extraction de colorant et l'usage desdites plantes par la population de la zone d'étude. L'analyse de ce tableau montre que les familles des Caesalpiniaceae (12,5\%), Combretaceae, Fabaceae, Anacardiaceae (10\%), Poaceae, Cochlospermaceae, Méliaceae et Mimosaceae (5\%), sont dominantes et représentent à elles seules $62,5 \%$ des espèces recensées. Ces familles d'espèces végétales pourraient servir comme guide intéressant d'orientation à la recherche des sources de molécules tinctoriales. Plus de $70 \%$ d'espèces recensées sont utilisées traditionnellement en textile et en teinture (teindre le corps et les objets fabriqués dans l'artisanat).

\section{Parties de la plante utilisée comme source de colorants}

Il ressort de cette enquête ethnobotanique que diverses parties de ces plantes tinctoriales, dont les écorces, feuilles, fleurs, fruits, graines, noix, gommes, et les parties souterraines (racine et rhizome) sont récoltées et utilisées dans la préparation de colorants. En effet, les écorces de tronc ont été utilisées dans 14 des 40 espèces répertoriées. Cette partie de la plante est la plus sollicitée. Les feuilles et rameaux feuillés sont utilisés dans 10 espèces. Viennent ensuite les racines ( 5 espèces), les fruits (5 espèces), les fleurs (3 espèces), les gommes (3 espèces).

\section{Modes d'extraction des colorants}

Divers modes d'extraction des colorants dont la décoction, le broyage, l'infusion, la macération, le pétrissage, le pilage et la fermentation ont été enregistrés. Toutefois, la décoction est la technique la plus sollicitée (50\%) par les artisans pour la production de colorants naturels (Tableau 2). En outre, certaines des personnes enquêtées, particulièrement les tradipraticiens, disent avoir préconisé cette technique pour l'extraction de certains principes actifs pour les traitements des maladies et infections.

\section{Résultats du criblage phytochimique}

Les résultats des tests phytochimiques effectués sur les extraits des vingt espèces végétales les plus cités sont mentionnés dans le Tableau 3. Des tannoïdes, des tanins catéchiques, des tanins galliques, des flavonoïdes, des anthocyanes, des leuco anthocyanes, des quinones, des coumarines, des alcaloïdes, des stérols et polyterpènes ont été détectés. L'analyse de ces résultats a révélé que plus de la moitié des plantes retenues ont montré la présence des différents groupes chimiques recherchés. Les tannoïdes et/ou les polyphénols constituent le groupe le plus fréquemment retrouvé dans ces espèces. Ils sont présents dans 19 (soit 95\%) des 20 espèces sélectionnées, dont 15 (soit 75\%) renfermant des tanins catéchiques et 12 $(60 \%)$ des tanins galliques (Figure 1). Pour le groupe des flavonoïdes, ils sont présents respectivement dans 15 plantes. Pour les groupes de coumarines, alcaloïdes, leur présence est détectée dans 13 de ces plantes. Les anthocyanes et leuco anthocyanes sont respectivement révélés dans 11 et 12 de ces plantes. Quant aux polyterpènes, les stérols et les quinones, leur présence dans les extraits aqueux n'a pas du tout été détectée. Pour des raisons de solubilité et affinité avec les solvants, des extraits éthériques ont été préparés, ce qui a permis de les détectés plutôt dans les extraits de l'éther de pétrole que dans ceux aqueux. Ces derniers sont présents dans 10 de ces plantes. La présence de tous ces groupes de composés chimiques dans ces plantes explique et/ou justifie la couleur observée dans les extraits. 
Tableau 2: Résultats de l'inventaire des plantes tinctoriales recensées.

\begin{tabular}{|c|c|c|c|c|c|c|c|c|c|}
\hline Familles & Genres-Espèces & $\begin{array}{l}\text { État } \\
\text { plante }\end{array}$ & Noms locaux & $\begin{array}{c}\text { Parties } \\
\text { utilisées }\end{array}$ & $\begin{array}{c}\text { Type } \\
\text { croissance }\end{array}$ & $\begin{array}{c}\text { Mode } \\
\text { extraction }\end{array}$ & $\begin{array}{l}\text { Couleur } \\
\text { d'extrait }\end{array}$ & Usage & $\begin{array}{c}\text { Nombre } \\
\text { de fois cité }\end{array}$ \\
\hline \multirow[t]{4}{*}{ Anacardiaceae } & $\begin{array}{l}\text { Lannea microcarpa Engl. et } \\
\qquad K r\end{array}$ & $\mathrm{~Sv}$ & $\begin{array}{l}\text { Faroun moutané, } \\
\text { Falunfa }\end{array}$ & ET & Arbre & Décoction & Rouge & Textile & 11 \\
\hline & Lannea acida $L$ & $\mathrm{~Sv}$ & Tamarza & ET & arbre & Décoction & Beau rouge & Teinture & 10 \\
\hline & Mangifera indica $L$. & $\mathrm{Sv}, \mathrm{Cul}$ & Màngwàrò & $\mathrm{Fe}$ & Arbre & Décoction & Colorant Jaune & Textile & 7 \\
\hline & $\begin{array}{c}\text { Sclerocarya birrea }(\text { A. Rich }) \\
\text { Hochst }\end{array}$ & $\mathrm{Sv}$ & Daniya , Diney & ET & Arbre & Décoction & Rouge Brun & Artisanat & 12 \\
\hline Boraginaceae & $\begin{array}{l}\text { Arnebia hispidissima } \\
\text { (Sieber Ex Lehm.) Dc }\end{array}$ & $\mathrm{Sv}$ & $\begin{array}{l}\text { Jinin mutum, } \\
\text { Jina-Jina }\end{array}$ & $\mathrm{Rc}$ & Herbe & Décoction & Rouge Violacée & $\begin{array}{c}\text { Textile } \\
\text { Teindre le corps } \\
\end{array}$ & 3 \\
\hline Burseraceae & $\begin{array}{c}\text { Commiphora africana (A. } \\
\text { Rich) Engl. }\end{array}$ & $\mathrm{Sv}$ & $\begin{array}{l}\text { Dâshi, Iskitchi } \\
\text { Korombé, }\end{array}$ & ET & Arbuste & Macération & Rouge & $\begin{array}{c}\text { Textile } \\
\text { Vannerie } \\
\end{array}$ & 2 \\
\hline \multirow[t]{5}{*}{ Cesalpiniaceae } & Senna occidentalis L. & $\mathrm{Sv}, \mathrm{Cul}$ & $\begin{array}{c}\text { Kinkiliba, } \\
\text { Raydoré } \\
\text { Sanga-Sanga }\end{array}$ & $\mathrm{Fe}+\mathrm{ram}$ & Herbe & Décoction & Bleue d'indigo & Teinture & 2 \\
\hline & Tamarindus indica $L$. & $\mathrm{Sv}$ & $\begin{array}{l}\text { Tsamia, } \\
\text { Bôsey, }\end{array}$ & $\mathrm{Fe}, \mathrm{ET}$ & Arbre & Decoction & Brune- rouge & Teinture & 7 \\
\hline & Cassia sieberana DC. & $\mathrm{Sv}$ & Malga, Sissan, & $\mathrm{Rc}$ & Arbre & Maceration & Noire & Teinture & 2 \\
\hline & $\begin{array}{c}\text { Piliostigma reticulatum } \\
\text { (DC) Hochst. }\end{array}$ & $\mathrm{Sv}$ & Kalgo, Kossey & ET & Arbre & Décoction & Rouge & $\begin{array}{l}\text { Vannerie, } \\
\text { Poterie }\end{array}$ & 5 \\
\hline & $\begin{array}{c}\text { Detarium microcarpum G. } \\
\text { et Perr. }\end{array}$ & $\mathrm{Sv}$ & Taura, Fantou & ET & Arbre & Décoction & Rouge- noire & $\begin{array}{l}\text { Teinture } \\
\text { Textile }\end{array}$ & 13 \\
\hline Capparidaceae & Crateva religiosa Sieber. & $\mathrm{Sv}$ & $\begin{array}{c}\text { Gudé /Léléo, } \\
\text { Angedudu }\end{array}$ & Fe, Go & Arbre & $\begin{array}{l}\text { Broyage et } \\
\text { Infusion }\end{array}$ & Jaune & $\begin{array}{c}\text { Textile } \\
\text { Vannerie }\end{array}$ & 1 \\
\hline \multirow[t]{2}{*}{ Combretaceae } & $\begin{array}{c}\text { Combretum nigricans Lepr. } \\
\text { Var. Elliotii (Engl et D) } \\
\text { Aub. } \\
\end{array}$ & $\mathrm{Sv}$ & $\begin{array}{l}\text { Tsiriry, Dagéra } \\
\text { Déli }\end{array}$ & Go & arbre & $\begin{array}{l}\text { Infusion et } \\
\text { décoction }\end{array}$ & Brune & Textile & 2 \\
\hline & Anogeissus leiocarpus (Dc.) & $\mathrm{Sv}$ & Maréké/ & $\mathrm{Fe}, \mathrm{ET}$ & Arbre & Décoction & Jaune, orange & Teinture (les & 14 \\
\hline
\end{tabular}


M. SOULEY KALLO et al. / Int. J. Biol. Chem. Sci. 12(2): 867-883, 2018

\begin{tabular}{|c|c|c|c|c|c|c|c|c|c|}
\hline & Guill. et Perr. & & N'Gongo & & & $(+$ Potasse $)$ & Brune & $\begin{array}{l}\text { étoffes, } \\
\text { Cuirs...) }\end{array}$ & \\
\hline & $\begin{array}{c}\text { Combretum Glutinosum } \\
\text { Perr. }\end{array}$ & $\mathrm{Sv}$ & $\begin{array}{c}\text { Kántákàráá } \\
\text { Taramnya, } \\
\text { Kokorbé }\end{array}$ & Fr, Rc & Arbuste & Macération & Jaune brunâtre & Textile & 3 \\
\hline & $\begin{array}{c}\text { Terminalia avicen noides } \\
\text { G. et Perr. }\end{array}$ & $\mathrm{Sv}$ & $\begin{array}{c}\text { Bawshi/ Farkg } \\
\text { Hanga }\end{array}$ & $\mathrm{Fe}$ & Arbre & $\begin{array}{l}\text { Pilage et } \\
\text { Infusion }\end{array}$ & Jaune & Teinture & 4 \\
\hline Convolvulaceae & $\begin{array}{l}\text { Merremia pinnata (Hochst.) } \\
\text { Hallier. }\end{array}$ & $\mathrm{Sv}$ & $\begin{array}{l}\text { Gamon Fulani } \\
\text { Kongo Zâra }\end{array}$ & $\mathrm{PE}$ & Herbe & Décoction & Noire & Teinture & 2 \\
\hline \multirow[t]{2}{*}{ Cochlospermaceae } & $\begin{array}{c}\text { Cochlospermum planchonii } \\
\text { Hook. }\end{array}$ & $\mathrm{Sv}$ & $\begin{array}{c}\text { Balangandé } \\
\text { Samaraye }\end{array}$ & $\mathrm{Rc}$ & Arbuste & $\begin{array}{l}\text { Macération } \\
\text { Infusion }\end{array}$ & Jaune & Textile & 13 \\
\hline & $\begin{array}{c}\text { Cochlospermum tinctorium } \\
\text { A. Rich }\end{array}$ & $\mathrm{Sv}$ & $\begin{array}{c}\text { Kukur, Kota } \\
\text { Rawaya }\end{array}$ & Rc & Herbe & Macération & Jaune- orangé & Textile & 12 \\
\hline \multirow{4}{*}{ Fabaceae } & Cassia alata $L$ & $\mathrm{~Sv}$ & Hilasko & $\mathrm{Rc}$ & Arbuste & Décoction & Noire & Tatouage & 1 \\
\hline & Indigofera tinctoria $L$ & $\mathrm{~Sv}, \mathrm{Cul}$ & Bâba/ Sîni & $\mathrm{Fe}+\mathrm{ram}$ & Arbuste & $\begin{array}{c}\text { Infusion, } \\
\text { Fermentation }\end{array}$ & Bleue variable & $\begin{array}{c}\text { Teinture (les } \\
\text { étoffes, Raphias) }\end{array}$ & 16 \\
\hline & $\begin{array}{l}\text { Lonchocarpus cyanescens } \\
\text { (Schum. \& Thonn.) Benth }\end{array}$ & $\mathrm{Sv}$ & $\begin{array}{l}\text { Baban } \\
\text { talaki }\end{array}$ & $\mathrm{Fe}, \mathrm{Br}$ & $\begin{array}{l}\text { Arbuste } \\
\text { grimpant }\end{array}$ & Infusion & Bleue variable & $\begin{array}{c}\text { Teinture (les } \\
\text { étoffes, Raphias) }\end{array}$ & 3 \\
\hline & Delonix regia (Boj) Raf. & $\mathrm{Sv}, \mathrm{Cul}$ & flamboyant & $\mathrm{Fl}$ & & Macération & Rouge & $\begin{array}{c}\text { Teinture } \\
\text { Vannerie }\end{array}$ & 5 \\
\hline Lythraceae & Lawsonia inermis $L$. & $\mathrm{Cul}$ & lallé & $\mathrm{Fe}$ & Arbuste & $\begin{array}{l}\text { Macération + } \\
\text { cendre }\end{array}$ & Orange et noire & $\begin{array}{c}\text { Cosmetique, } \\
\text { tatouage }\end{array}$ & 12 \\
\hline Malvaceae & Hibiscus sabdariffa $L$. & $\mathrm{Sv}, \mathrm{Cul}$ & Yakwa Bissap & $\mathrm{Fl}$ & Herbe & Macération & Rouge & $\begin{array}{l}\text { Cosmétique } \\
\text { Alimentaire }\end{array}$ & 16 \\
\hline \multirow[t]{2}{*}{ Meliaceae } & Azadirachta indica A. Juss. & $\mathrm{Sv}, \mathrm{Cul}$ & Dogon yaro & Go & Arbre & $\begin{array}{l}\text { Infusion et } \\
\text { Macération }\end{array}$ & Rouge & Teinture & 5 \\
\hline & $\begin{array}{c}\text { Khaya senegalensis (Desv.) } \\
\text { A. Juss. }\end{array}$ & $\mathrm{Sv}, \mathrm{Cul}$ & $\begin{array}{l}\text { Maddatchi } \\
\text { farey }\end{array}$ & ET & Arbre & $\begin{array}{l}\text { Décoction } \\
\text { Infusion }\end{array}$ & Rouge orangée & Teinture & 9 \\
\hline Mimosaceae & $\begin{array}{l}\text { Parkia biglobosa (Jacq.) } \\
\text { Benth. }\end{array}$ & $\mathrm{Sv}$ & Dorowa, Dso & ET, Co & Arbre & $\begin{array}{l}\text { Pilage Et } \\
\text { Infusion }\end{array}$ & Brune, rouge & Teinture & 11 \\
\hline
\end{tabular}


M. SOULEY KALLO et al. / Int. J. Biol. Chem. Sci. 12(2): 867-883, 2018

\begin{tabular}{|c|c|c|c|c|c|c|c|c|c|}
\hline & Acacia nilotica (L.) Willd. & $\mathrm{Sv}$ & $\begin{array}{l}\text { Bagaruwa, } \\
\text { Baani, Jitti }\end{array}$ & ET, Fr & Arbre & Décoction & $\begin{array}{l}\text { Kaki, brune, } \\
\text { grise, noire }\end{array}$ & $\begin{array}{l}\text { Tannerie } \\
\text { artisanale }\end{array}$ & 11 \\
\hline Papilionaceae & Pterocarpus erinaceus Poir & $\mathrm{Sv}$ & Madobiy, Tôlo & ET, Bo & Arbre & $\begin{array}{l}\text { Décoction } \\
(+ \text { Potasse }) \\
\end{array}$ & Rouge, brune & Textile & 7 \\
\hline & Sorghum bicolor & $\mathrm{Cul}$ & Jad-dawa & $\mathrm{Gr}, \mathrm{Fe}$ & Arbuste & $\begin{array}{l}\text { Macération + } \\
\text { citron }\end{array}$ & Rouge & $\begin{array}{l}\text { Vannerie } \\
\text { (peaux) }\end{array}$ & 8 \\
\hline Poaceae & Sorghum spp & $\mathrm{Sv}, \mathrm{Cul}$ & Kemé, Karandafi & $\mathrm{Fe}$ & Herbe & $\begin{array}{l}\text { Macération + } \\
\text { citron }\end{array}$ & Rouge & $\begin{array}{l}\text { Vannerie, } \\
\text { Textile }\end{array}$ & 13 \\
\hline Punicaceae & Punica granatum $L$. & Cul & $\begin{array}{c}\text { Roumani, } \\
\text { Araman } \\
\end{array}$ & $\mathrm{Fl}, \mathrm{Fr}$ & Arbuste & $\begin{array}{l}\text { Décoction } \\
+ \text { alun } \\
\end{array}$ & $\begin{array}{l}\text { Jaune, brune, } \\
\text { rouge }\end{array}$ & Teinture & 5 \\
\hline Rubiaceae & $\begin{array}{c}\text { Sarcocephalus latifolias } \\
\text { (Sm.) Bruce. }\end{array}$ & $\mathrm{SV}$ & Igiya, kulukulu & $\mathrm{Rc}$ & Arbuste & Macération & Jaune foncé & Tannerie Textile & 9 \\
\hline Rubiaceae & & $\mathrm{Sv}$ & $\begin{array}{l}\text { Gaudé } \\
\text { Komdi, }\end{array}$ & $\mathrm{Fr}$ & Arbre & Macération & Noire & Tatouage & 7 \\
\hline Scrofulariaceae & $\begin{array}{c}\text { Striga hermontheaca (Del.) } \\
\text { Benth. }\end{array}$ & $\mathrm{Sv}$ & $\begin{array}{l}\text { Gáúgáí, } \\
\text { Mâli, } \\
\end{array}$ & $\mathrm{PE}$ & Herbe & Décoction & Noire & Teinture & 11 \\
\hline Solanaceae & Capsicum frutescens $L$ & $\mathrm{~Sv}, \mathrm{Cul}$ & Tsààkíí, Tachi & Fr & Arbuste & Pilage & Rouge & Alimentaire & 5 \\
\hline Sterculiaceae & $\begin{array}{c}\text { Cola nitida (Vent.) Schott \& } \\
\text { Endl }\end{array}$ & Cul & Goro, Ajauru & Noix & Arbre & Macération & Orange-Rouge & Teinture & 7 \\
\hline Verbénaceae & Vitex doninia Sweet & $\mathrm{Sv}$ & $\begin{array}{c}\text { Dhumnia, } \\
\text { Bôye }\end{array}$ & ET, Rc & Arbre & Décoction & $\begin{array}{l}\text { Rouge Brune, } \\
\text { Noire }\end{array}$ & $\begin{array}{c}\text { Teinture ; } \\
\text { Poterie }\end{array}$ & 6 \\
\hline Zygophyllaceae & $\begin{array}{c}\text { Balanites aegyptiaca (L.) } \\
\text { Del. }\end{array}$ & $\mathrm{Sv}$ & Aduwa, Garbey & ET, Fr & Arbre & Macération & Jaune & Teinture & 5 \\
\hline Chrysobalanaceae & $\begin{array}{c}\text { Neocarya macrophylla } \\
\text { (Sabine) Prance ex F. White }\end{array}$ & $\mathrm{Sv}$ & Gawasa, gamsa & ET & Arbre & Décoction & Rouge à brune & Teinture & 2 \\
\hline
\end{tabular}


M. SOULEY KALLO et al. / Int. J. Biol. Chem. Sci. 12(2): 867-883, 2018

Tableau 3 : Résultats de criblage phytochimique.

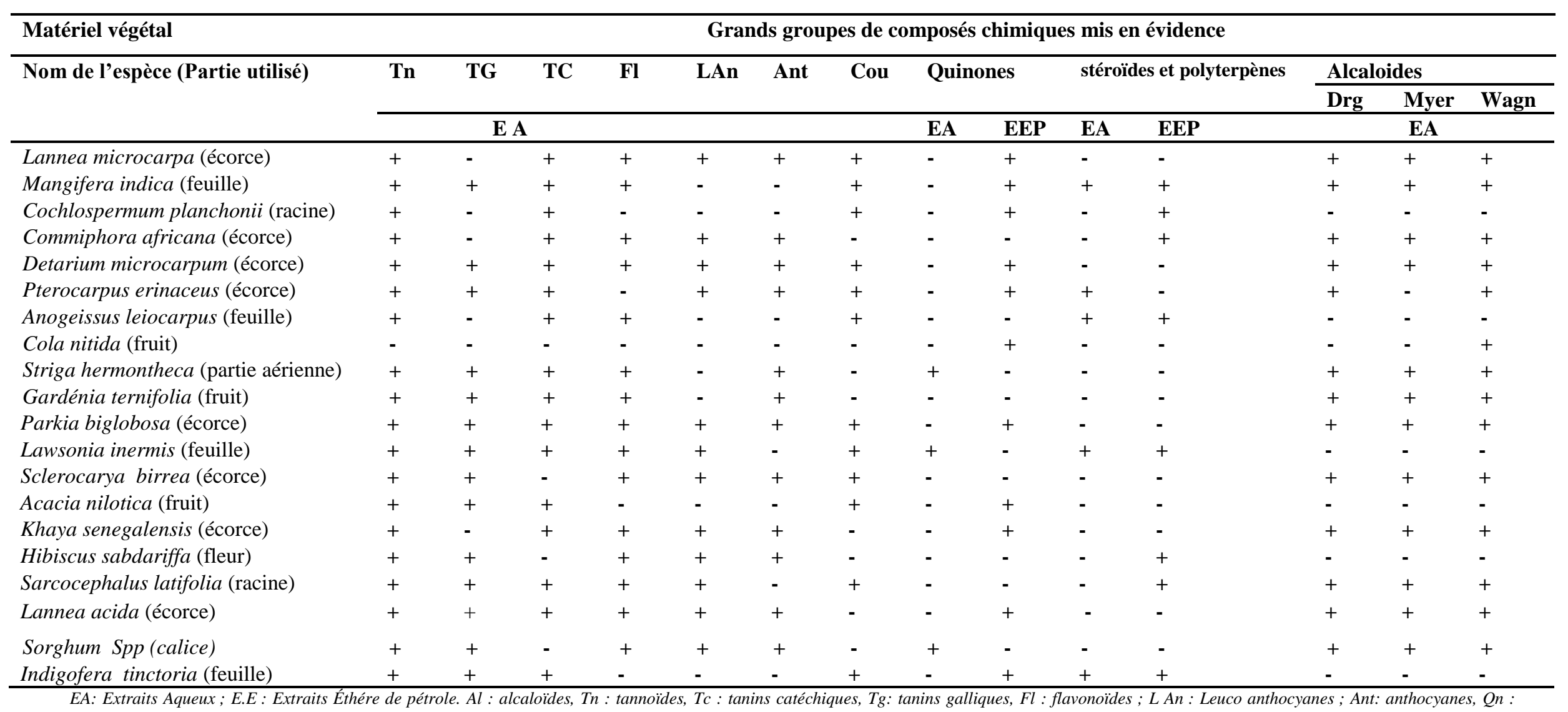

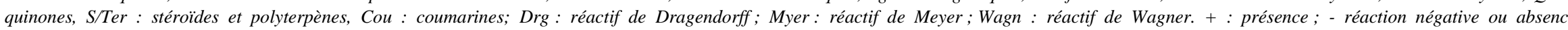




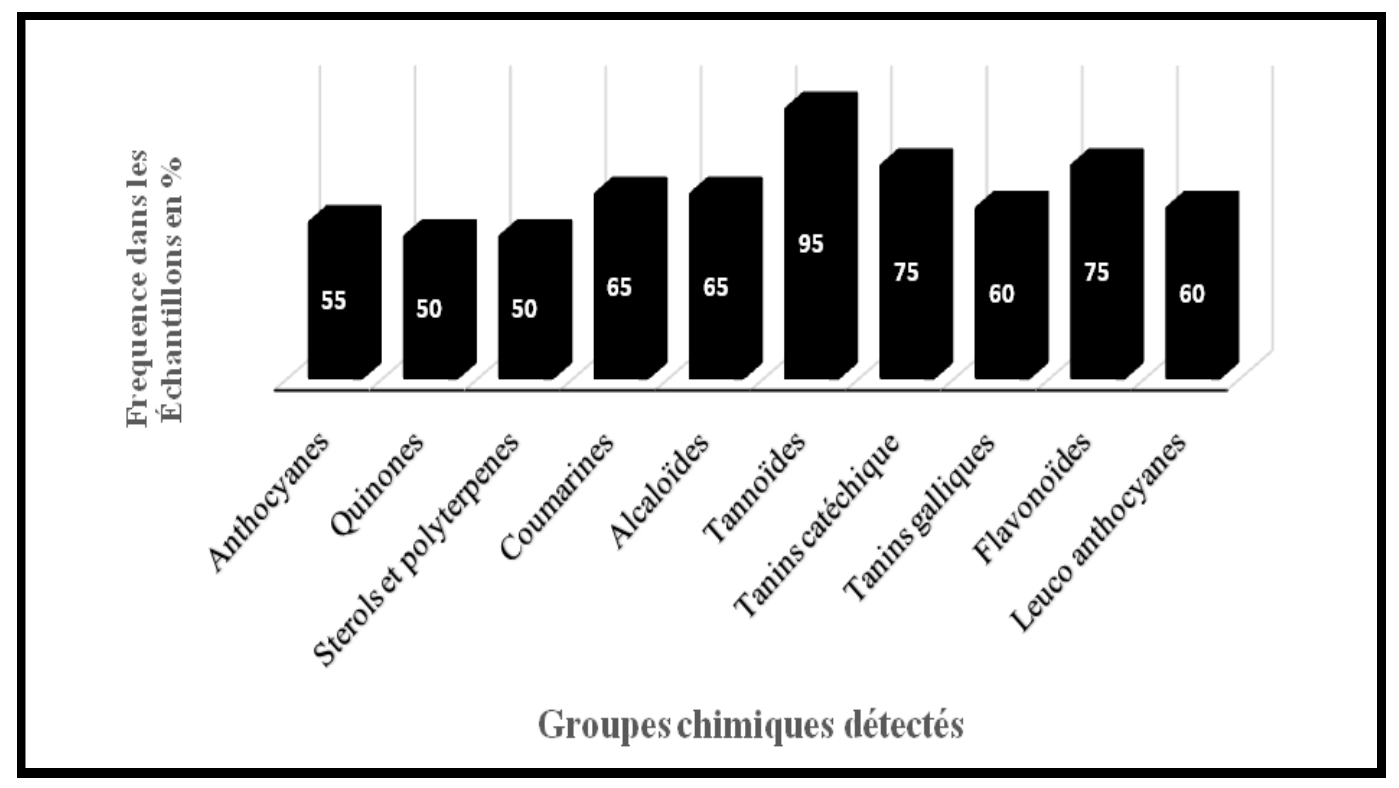

Figure 1: Fréquence des groupes chimiques dans les espèces testées.

\section{DISCUSSION}

Les plantes recensées ont été principalement utilisées en maroquinerie, poterie, cosmétique et esthétique, mais aussi dans la teinture des cordes, des nattes, de la céramique et autres parures traditionnelles. Leur raison d'utilisation traditionnelle comme colorants est due en partie à la teinture qu'elles laissent au toucher, à leur aspect, pour des raisons culturelles ou pour des besoins d'esthétiques. Ces résultats sont similaires à ceux trouvés par Mabika et al. (2013) en Afrique centrale et ceux de Sore et al. (2012) au Burkina Faso. Certaines d'entre elles ont été déjà citées et utilisées comme plantes tinctoriales (Siva, 2007; Sore et al., 2012; Mabika et al., 2013). D'autres ont été étudiées pour des raisons thérapeutiques comme source des principes actifs dans le traitement de certaines maladies et infections (Odeja et al., 2014; Koffi et al., 2015). En ce qui concerne les parties à principe tinctorial de la plante, l'usage des écorces et des feuilles est plus sollicité. Ce résultat est en accord avec celui trouvé par Mabika et al. (2013) en Afrique centrale où les écorces, les feuilles et les racines sont également les parties les plus utilisées. Par ailleurs, il a été remarqué, en ce qui concerne les propriétés tinctoriales, que le mode de préparation est fonction de l'usage tinctorial. Ainsi, la décoction est la technique d'extraction la plus utilisée. Ce résultat semble confirmer celui de N'Guessan et al. (2009) qui montre que la décoction est majoritairement utilisée avec $42,30 \%$ des cas. Selon Cardon et al. (2010) les aspects tinctoriaux sont plus perceptibles avec l'ajout d'autres substances telles que le jus de citron (apport d'acide), la potasse et la cendre (apport de base) qui pourraient provoquer une modification structurale des chromophores présents dans la molécule du colorant. Les plantes recensées sont également source de principes actifs et leurs recettes et indications thérapeutiques ont été brièvement indiquées dans des travaux antérieurs (Zerbo et al., 2012; Odeja et al., 2014; Koffi et al., 2015; Béné et al., 2016).

Par ailleurs, le criblage phytochimique a révélé que les écorces du tronc des espèces, dont Lannea microcarpa Engl. et K. Krause (Anacardiaceae), Sclerocarya birrea (A. Rich) Höchst (Anacardiaceae), Detarium microcarpum Guill. et Perr. (Caésalpiniaceae), Khaya senegalensis (Desv.) A. Juss (Méliaceae), Parkia 
biglobosa (Jacq.) Benth (Mimosaceae), Pterocarpus erinaceus Poire (Papilionaceae) et Commiphora africana (A. Rich) Engl. (Burseraceae) sont riches en tanins, anthocyanines, leuco anthocyanes, coumarines et flavonoïdes. Ces plantes peuvent alors servir de sources potentielles desdits pigments. Toutefois, les tanins sont présents dans les extraits des écorces de ces plantes en quantité appréciable, ce qui suggère que les écorces desdites plantes peuvent également être utilisées dans les tanneries. Ces résultats sont en accord avec ceux obtenus dans les travaux antérieurs effectués sur certaines de ces espèces (Millogo-Kone et al., 2007; Sereme et al., 2008; Millogo-Kone et al., 2008; Fagbohoun, 2014). D'autres auteurs ont montré que les végétaux tannants renferment, en plus des substances tinctoriales, de nombreuses substances actives, ce qui explique leur utilisation accrue en médecine traditionnelle par la population locale (Amonkan et al., 2010).

Ces substances naturelles (tanins, anthocyanines, anthocyanes, coumarines et flavonoïdes) sont dotées des couleurs diversifiées (rouge, violette, jaune, orange, brune, bleue...). Elles sont prometteuses dans la collecte des radiations solaires, ce qui permettrait leur large utilisation dans les cellules solaires à pigments photosensibles. En plus, ces pigments végétaux ont, grâce à leurs groupes fonctionnels d'ancrage (hydroxyle, carboxyle), la capacité de se lier aisément de manière covalente avec les nanoparticules $\mathrm{du}$ semi-conducteur (Olounladé et al., 2011; Calogero et al., 2012; Narayan, 2012). De tels pigments constitueraient des bons candidats dans l'élaboration des cellules solaires à pigments photosensibles.

En outre, les polyterpènes et les stéroïdes sont détectés de manière importante dans les extraits éthériques d'espèces de Mangifera indica L. (Anacardiaceae), Cochlospermum planchonii hook (Cochlospermaceae), Commiphora africana (A. Rich) Engl (burseraceae), Anogeissus leiocarpus (DC.) Guill. et Perr.
(Combretaceae), Striga hermontheca (Del.) Benth (Scrofulariaceae) Sarcocephalus latifolia, (sm) Bruce (Rubaceae), Sorghum spp (Poaceae), Lawsonia inermis $\mathrm{L}$. (Lythraceae) et Indigofera tinctoria $L$ (Fabaceae). Les polyterpènes font partie de la famille de caroténoïdes, pigments jaune, orange et rouge rencontrés chez les végétaux supérieurs (Bartley et al., 1995; Mortensen, 2006). Ils ont également fait l'objet des nombreuses investigations dans le domaine de l'énergie notamment dans les cellules photovoltaïques à colorants (Yamazaki et al., 2007). Les alcaloïdes sont également présents dans les espèces Cola nitida (Vent.) Schott \& Endl (Sterculiaceae), Commiphora africana (A. Rich) Engl. (Burseraceae), Sarcocephalus latifolia, (sm) Bruce (Rubaceae), Khaya senegalensis (Desv.) A. Juss (Méliaceae) et Gardenia ternifolia K. Schum (Rubaceae).

La phytochimie des alcaloïdes a été beaucoup étudiée non seulement pour examiner et évaluer leurs propriétés thérapeutiques (Badiaga, 2011; Seck et al., 2015), mais aussi pour leurs propriétés tinctoriales dues à leur capacité à pigmenter la matière (Mabika et al., 2013). Ainsi, en plus des propriétés thérapeutiques, de nombreux travaux (Oliver-Bever, 1983; Hoekou et al., 2012; N'draman-Donou et al., 2015) montrent que les alcaloïdes quaternaires souvent présents dans les plantes sous forme de sels (chlorure, sulfate principalement) pouvaient être des principes tinctoriaux des colorants jaunes basiques. Cela semble confirmer les résultats de criblage phytochimique de Badiaga (2011) et Koko et al. (2011) effectué sur Sarcocephalus latifolia, (sm) Bruce(Rubaceae). De nombreuses études attribuent aux caroténoïdes, flavonoïdes, anthocyanes, tanins, composés quinoniques et alcaloïdes quaternaires des propriétés tinctoriales (Sereme et al., 2008; Cardon et al., 2010; Mabika et al., 2013). Beaucoup de travaux ont porté sur la phytochimie des plantes et des résultats similaires ont été obtenus (Alilou et al., 2014; Koffi et al., 2015). La présence de ces familles chimiques dans les vingt espèces sélectionnées dans cette étude 
fait de ces dernières des sources potentielles de colorants naturels. Leur utilisation traditionnelle comme colorants dans l'artisanat, cosmétiques, maroquinerie, poterie, cosmétique et esthétique trouve alors leur justification. Aujourd'hui, cette utilisation doit largement faire l'objet d'une extension vers d'autres nouvelles applications particulièrement dans les nouvelles technologies photovoltaïques pour la conversion de l'énergie solaire en électricité.

\section{Conclusion}

Cette étude a permis de réaliser, pour la première fois, une enquête ethnobotanique sur les plantes tinctoriales du Niger et d'évaluer les propriétés tinctoriales des vingt (20) plantes les plus utilisées traditionnellement comme colorant en textile, maroquinerie, cosmétique, vannerie et esthétique. Les tannoïdes, flavonoïdes, anthocyanes, leucoanthocyanes, quinones, alcaloïdes, coumarines, stérols et polyterpènes sont détectés dans ces plantes. Ces groupes chimiques constituent les principaux composés naturels responsables de la pigmentation chez les végétaux. La présence remarquable desdits composés chimiques fait doter ces plantes des propriétés tinctoriales. Elles constituent alors des matières premières pour la production de colorants naturels d'origine végétale. Ces colorants pourraient être valorisés dans les technologies nouvelles photovoltaïques, notamment comme sensibilisateur dans la conversion de l'énergie solaire en électricité.

\section{CONFLITS D'INTÉRÊTS}

Tous les auteurs déclarent qu'il n'y a aucun conflit d'intérêts pour la publication de cet article.

\section{CONTRIBUTION DES AUTEURS}

MMK a contribué aux différentes collectes des échantillons sur les différents sites d'études, à l'analyse des échantillons et à la rédaction du manuscrit. JS. a apporté sa contribution dans la rédaction scientifique et dans la correction du projet d'article. AAM a apporté lui aussi des commentaires sur le document. IMM a été utile pour l'identification des plantes et a participé à l'écriture et à l'amélioration du manuscrit. KI a apporté sa contribution dans les travaux relatifs au criblage phytochimique et la caractérisation des colorants. Enfin, AR a proposé le thème, a encadré le travail et a finalisé la rédaction du manuscrit.

\section{REMERCIEMENTS}

Nos remerciements au professeur de chimie Organique, M. Khalid IKHIRI de l'Université Abdou Moumouni de Niamey Niger qui est toujours actif pour nous accompagner dans la recherche scientifique. Par ailleurs, nous remercions Monsieur Arzika Tanimoune, technicien au département de Biologie de la Faculté des Sciences et Techniques, Université Abdou Moumouni de Niamey (Niger) pour sa contribution.

\section{REFERENCES}

Alilou H, Bencharki B, Hassani L I, Barka N. 2014. Screening phytochimique et identification spectroscopique des flavonoïdes d'Asteriscusb graveolens subsp. Odorus. Afrique Science : Revue Internationale des Sciences et Technologie, 10(3) : 316-328.

Amonkan A, Konan A, Kouakou L, Bouafou G, Bleyere M, Ahui M, Zannou V, Ouattara H, Datte J, Kati-Coulibaly S. 2010. Criblage phytochimique et effets d'un extrait aqueux de feuilles de Ficus exasperata Vahl. 1805 (Moraceae) sur la pression artérielle et l'activité contractile du cœur chez les mammifères. International Journal of Biological and Chemical Sciences, 4(3): 681-691.

Badiaga M, 2011. Etude ethnobotanique, phytochimique et activités biologiques de Nauclea latifolia Smith, une plante médicinale africaine récoltée au Mali,.Doctorat d'Université, Université Blaise Pascal-Clermont-Ferrand II, 184 p.

Bakasso S, 2009. Etudes phytochimiques et potentialités biologiques de cinq espèces 
d'Indigofera (Fabaceae) utilisées en médecine traditionnelle au Burkina Faso, Thèse unique, Université de Ouagadougou, Burkina Faso.

Bartley GE, Scolnik PA. 1995. Plant carotenoids: pigments for photoprotection, visual attraction, and human health. The Plant Cell, 7(7): 1027-1038.

Bekro Y-A, Mamyrbekova J, Boua BB, Bi F T, Ehile EE. 2007. Étude ethnobotanique et screening phytochimique de Caesalpinia benthamiana (Baill.) Herend. et Zarucchi (Caesalpiniaceae). Sciences \& Nature, 4(2): 217-225.

Béné K, Camara D, Fofie NGBY, Kanga Y, Yapi AB, Yapo YC, Ambe SA, Zirihi G N. 2016. Étude ethnobotanique des plantes médicinales utilisées dans le Département de Transua, District du Zanzan (Côte d'Ivoire). Journal of Animal \&Plant Sciences, 27(2): 42304250, ISSN 2071-7024.

Boye A, Barku V, Amoateng P, Mbroh L. 2014. Anti-nociceptive and antioxidant activities of an aqueous leaf extract of Corchorus olitorius L.(Tiliaceae). International Journal of Biological and Chemical Sciences, 8(6): 2395-2406. doi: http://dx.doi.org/10.4314/ijbcs.v8i6.3

Calogero G, Yum J-H, Sinopoli A, Di Marco G, Grätzel M, Nazeeruddin M K. 2012. Anthocyanins and betalains as lightharvesting pigments for dye-sensitized solar cells. Solar Energy, 86(5): 15631575, doi: http://dx.doi.org/10.1016/ j.solener.2012.02.018.

Campêlo LML, de Almeida AAC, de Freitas RLM, Cerqueira GS, de Sousa GF, Saldanha GB, Feitosa CM, de Freitas RM. 2011. Antioxidant and antinociceptive effects of Citrus limon essential oil in mice. BioMed Research International, $\quad \mathbf{1}$ : $1-8, \quad$ doi: 10.1155/2011/678673.

Cardon D, Cabalion P, Blanc B, BoulangerPenduff M, Hnawia E, Nowik W. 2010. Plantes tinctoriales des traditions et sociétés du Pacifique: de la sauvegarde des savoirs aux nouvelles perspectives d'application. Ethnopharmacologia, 46(1): 17-23, doi: halshs-00675054

Ciulei I. 1982, Practical manuals on the industrial utilization of chemical and aromatic plants. In Methodology for Analysis of Vegetable Drugs ( $1^{\text {st }}$ Edn). Ministry of Chemical Industry: Bucharest.

Dohou R, Yamni K, Tahrouch S, Hassani L I, Badoc A, Gmira N. 2003. Screening phytochimique d'une endémique iberomarocaine, Thymelaea lythroides. Bulletin-Société de Pharmacie de Bordeaux, 142(1/4): 61-78.

Fagbohoun L. 2014. Etude chimique de colorants naturels et matériaux résineux traditionnels au Bénin dans le domaine artisanal, Université d'Avignon, 296 pages.

Gnahoué G, Béné K, Coulibaly K, 2015. Etude botanique, screening phytochimique et activité anticandidosique in vitro de pycnanthus angolensis

(WELW.)

WARB.(MYRISTICACEAE).

European Scientific Journal, 11(36), ISSN: 1857-7431.

Hoekou YP, Batawila K, Gbogbo KA, Karou DS, Ameyapoh Y, Souza C. 2012. Evaluation des propriétés antimicrobiennes de quatre plantes de la flore togolaise utilisées en médecine traditionnelle dans le traitement des diarrhées infantiles. International Journal of Biological and Chemical Sciences, 6(6): 3089-3097, doi: http://dx.doi.org/10.4314/ijbcs.v6i6.10

Kini F, Saba A, Ouedraogo S, Tingueri B, Sanou G, Guissou I. 2008. Potentiel nutritionnel et thérapeutique de quelques espèces fruitières «sauvages» $\mathrm{du}$ Burkina Faso. Pharmacopée et Médecine Traditionnelle Africaine, 15(1): 32 - 35, doi: http://www.cnrst.bf/. Koffi A, Bla K, Yapi H, Bidie A, Djaman A. 2015. Phytochemical Screening of Some Medicinal Plants in Côte D'ivoire and Evaluation of their Extraction Efficiency. International Journal of 
Pharmacognosy and Phytochemical Research, 7(3): 563-569.

Koko IKD, Djego J, Gbenou J, HounzangbéAdoté S, Sinsin B. 2011. Etude phytochimique des principales plantes galactogènes et emménagogues utilisées dans les terroirs riverains de la Zone cynégétique de la Pendjari. International Journal of Biological and Chemical Sciences, 5(2): 618 - 631.

Mabika A M, Loumpangou C N, Agnaniet H, Moutsamboté J, Ouamba J. 2013. Les plantes tinctoriales d'Afrique Centrale: Enquête ethnobotanique et screening phytochimique. Journal of Applied Biosciences, 67(1): 5236-5251.

MacFoy C. 2004. Ethnobotany and sustainable utilization of natural dye plants in Sierra Leone. Economic Botany, 58(sp1): S66-S76, doi: https://doi.org/10.1663/0013-

0001(2004)58[S66:EASUON]2.0.CO;2.

Millogo-Kone H, Guissou I, Nacoulma O, Traore A. 2007. Antimicrobial effects of the stem bark extracts of Parkia biglobosa (jacq.) Benth. on Shigellae. African Journal of Traditional, Complementary and Alternative Medicines, 4(4): 392-396.

Millogo-Kone H, Guissou I, Nacoulma O, Traore A. 2008. Comparative study of leaf and stem bark extracts of Parkia biglobosa against enterobacteria. African Journal of Traditional, Complementary and Alternative Medicines, 5(3): 238-243.

Młodzińska E. 2009. Survey of plant pigments: molecular and environmental determinants of plant colors. Acta Biologica Cracovienca Series Botanica, 51(1): 7-16.

Mortensen A. 2006. Carotenoids and other pigments as natural colorants. Pure and Applied Chemistry, 78(8): 1477-1491, doi: 10.1351/pac200678081477.

N'draman-Donou E, Fofié Y, Adjambri E, Mélèdje MF. Sawadogo D. 2015. Caractérisation et évaluation in vitro de l'effet antifalcimiant des graines de Cajanus cajan (Fabacées) sur les drépanocytes à Abidjan-Côte d'Ivoire. International Journal of Biological and Chemical Sciences, 9(5): 2300-2308, doi: http://dx.doi.org/10.4314/ijbcs.v9i5.4 N'Guessan K, Kadja B, Zirihi G, Traoré D, Aké-Assi L. 2009. Screening phytochimique de quelques plantes médicinales ivoiriennes utilisées en pays Krobou (Agboville, Côte-d'Ivoire). Sciences \& Nature, 6(1): 1-15.

Narayan M R, 2012. Dye sensitized solar cells based on natural photosensitizers. Renewable and Sustainable Energy Reviews, 16(1): 208-215, doi: https://doi.org/10.1016/j.rser.2011.07.14 8.

Odeja O, Obi G, Ogwuche C, Elemike E, Oderinlo O, 2014. Phytochemical screening, Antioxidant and Antimicrobial activities of Senna occidentalis (L.) leaves. International Journal of Herbal Medicine, 2(4): 2630.

Oliver-Bever B. 1983. Medicinal plants in tropical west Africa II. Plants acting on the nervous system. Journal of Ethnopharmacology, 7(1): 1-93, doi: https://doi.org/10.1016/03788741(83)90082-X.

Olounladé P, Hounzangbé-Adoté M, Azando E, Ha TT, Brunet S, Moulis C, Fabre N, Fouraste I, Hoste H, Valentin A. 2011. Etude in vitro de l'effet des tanins de Newbouldia laevis et de Zanthoxylum zanthoxyloïdes sur la migration des larves infestantes de Haemonchus contortus. International Journal of Biological and Chemical Sciences, 5(4): 1414-1422. doi: http://dx.doi.org/10.4314/ijbcs.v5i4.8.

Razafindrambao RS. 1973. Etude d'une plante médicinale malgache Buxus madagascarica Baill. et ses variétés, Thèse de 3è Cycle, 98p.

Saha S, Das P, Chakraborty AK, Sarkar S, Debbarma R. 2016. Fabrication of DSSC with nanoporous $\mathrm{TiO} 2$ film and Kenaf Hibiscus dye as sensitizer. International Journal of Renewable 
Energy Research (IJRER), 6(2): 620627.

Sangare M M, Sina H, Dougnon J, Bayala B, Ategbo J-M, Dramane K L. 2012. Etude ethnobotanique des plantes hépatotropes et de l'usage traditionnel de Gomphrena celosioides Mart.(Amaranthaceae) au Bénin. International Journal of Biological and Chemical Sciences, 6(6): 5008-5021.

doi: http://dx.doi.org/10.4314/ijbcs.v6i6.20.

Seck M, Sall C, Gueye PM, Seck I, Dioum M D, Lembachar Z, Guere R, Fall D, Fall M, Dieye TN. 2015. Etude de l'activité antifalcémiante d'extraits de racines de Leptadenia hastata Decne. (Asclepiadacae). International Journal of Biological and Chemical Sciences, 9(3): 1375-1383. doi: http://dx.doi.org/10.4314/ijbcs.v9i3.22.

Sereme A, Millogo-Rasolodimby J, Guinko S, Nacro M. 2008. Concentration en tanins des organes de plantes tannifères du Burkina Faso. Journal de la Société Ouest-Africaine de Chimie, 025(1): 5561.

Siva R. 2007. Status of natural dyes and dyeyielding plants in India. Current Science, 92(7): 916-925, doi: http://www.jstor.org/stable/24097672.

Sofowora A. 2010. Plantes Médicinales et Médecine Traditionnelle d'Afrique. : KARTHALA Editions.
Sore H, Hilou A, Sombie PAED, Compaore M, Millogo J, Nacoulma OG. 2012. Dyeing and Medicinal Plants Used in the Area of Mouhoun in Burkina Faso. Universal Journal of Environmental Research and Technology, 2(3): 110 118.

Tutak M, ACARG, Akman O. 2014. Natural dyeing properties of wool fabrics by pomegranate (Punica granatum) PEEL. Journal of Textile \& Apparel/Tekstil ve Konfeksiyon, 24(1): 81 - 85.

Udobi C, Onaolapo J. 2009. Phytochemical analysis and antibacterial evaluation of the leaf stem bark and root of the African locust bean (Parkia biglobosa). Journal of Medicinal Plants Research, 3(5): 338-344.

Yamazaki E, Murayama M, Nishikawa N, Hashimoto N, Shoyama M, Kurita O. 2007. Utilization of natural carotenoids as photosensitizers for dye-sensitized solar cells. Solar energy, 81(4): 512516.

doi: https://doi.org/10.1016/j.solener.2006.0 8.003 .

Zerbo P, Millogo RJ, Nacoulma O, Van Damme P. 2012. Plantes médicinales et pratiques médicales au Burkina Faso: cas des Sanan. Pharmacopée et Médecine Traditionnelle Africaine, 16(2): 1-13. 